\title{
Scalar glueball and meson spectroscopy in unquenched lattice QCD with improved staggered quarks
}

\author{
Eric B. Gregory, Alan C. Irving*, Craig C. McNeile, Steven Miller, Zbyszek \\ Sroczynski \\ Department of Mathematical Sciences, University of Liverpool \\ Liverpool, U.K. \\ E-mail: acieliv.ac.uk
}

\begin{abstract}
We present results of an exploratory study of singlet scalar states in unquenched QCD using both glueball and meson operators. Results for non-singlet non-strange scalar mesons are also presented. We use Asqtad improved staggered fermions and gauge configurations generated by the MILC collaboration at lattice spacings of .12 and $.09 \mathrm{fm}$. In this formulation, the glueball mass is not significantly different from the quenched value at finite lattice spacing. Significant taste violations are present in the scalar sector. At light quark masses, decay channels complicate the mass determinations. There is some evidence that the non-strange singlet meson lies below the non-singlet meson.
\end{abstract}

XXIIIrd International Symposium on Lattice Field Theory

25-30 July 2005

Trinity College, Dublin

\footnotetext{
* Speaker.
} 


\section{The scalar sector of QCD}

The scalar glueball is by now relatively well studied in quenched QCD. In full QCD with realistically light quarks it is not [1], 2] Simulations with moderate to heavy Wilson-like quarks show effects which may reflect poorly understood lattice artifacts rather than continuum physics [2]. UKQCD plans to use its new QCDOC dedicated supercomputer to create substantial data sets to complement the existing MILC configurations [3, 他 which will allow substantially higher statistics and moderate to light quark mases. Clearly, there are a number of serious challenges to be met. On the one hand light quark masses help one probe mixing but at the same time open up decay channels which complicate spectroscopy using current methods. Furthermore, the singlet sector may be precisely that sector where the staggered quark method is at its most vulnerable because of the differences in the implementation of valence and sea quarks and because of inadequately controlled taste-breaking effects in the sea.

\section{Glueballs}

Figure 11 shows effective masses for ensembles $C$ and $F$ of the MILC data sets [3, 4] indicated in Table 1 below. The comparison is made in physical units using measured values of $r_{1} / a$.

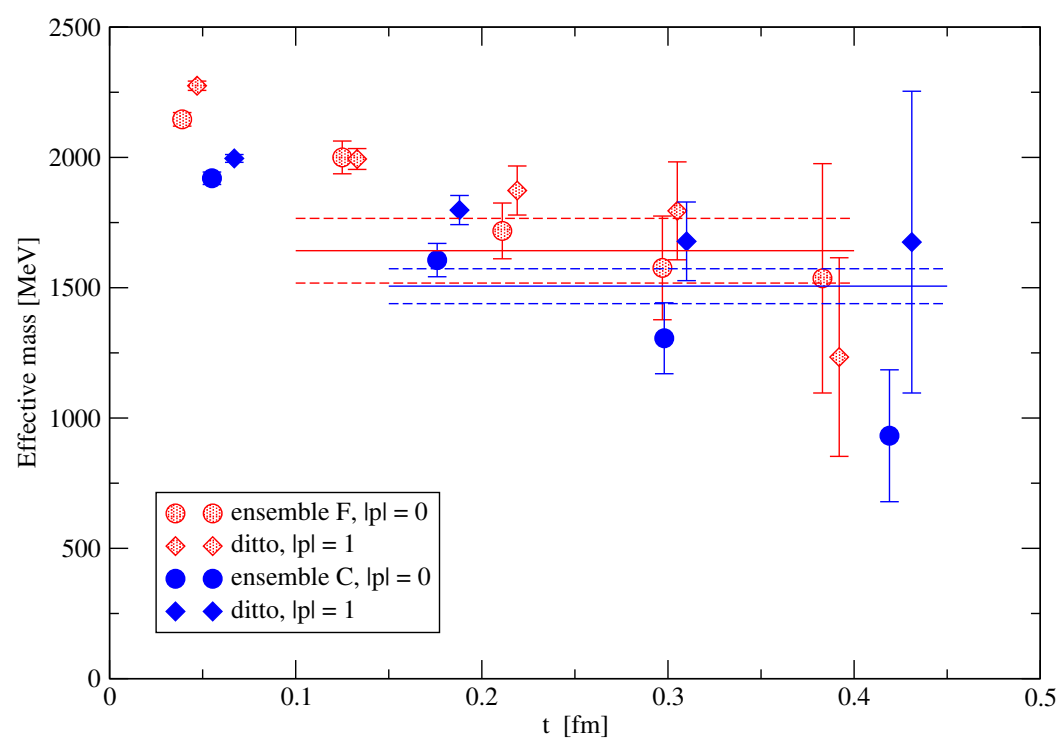

Figure 1: Effective masses using glueball operators.

Non-zero momentum operators are also used to help confirm the mass estimates. Horizontal lines indicate fitted values and errors. Figure 2 shows the results of such measurements superimposed on a compilation of previous quenched and unquenched results. We note that lattice artifacts appear smaller than for improved Wilson quarks with unimproved Wilson glue [2]. The current errors are clearly not yet small enough to resolve properly the experimental spectrum or quark mass dependence. 


\begin{tabular}{|c|c|c|c|c|c|}
\hline Ensemble & $\beta$ & $a m_{u, d}$ & $a m_{s}$ & $\mathrm{a}[\mathrm{fm}]$ & No. of configs \\
\hline $\mathrm{A}$ & 6.81 & 0.03 & 0.05 & 0.1191 & 564 \\
$\mathrm{~B}$ & 6.79 & 0.02 & 0.05 & 0.1196 & 483 \\
$\mathrm{C}$ & 6.76 & 0.01 & 0.05 & 0.1215 & 658 \\
$\mathrm{D}$ & 6.76 & 0.007 & 0.05 & 0.1209 & 448 \\
\hline $\mathrm{E}$ & 7.11 & 0.0124 & 0.031 & 0.0854 & 514 \\
$\mathrm{~F}$ & 7.09 & 0.0062 & 0.031 & 0.0860 & 505 \\
\hline
\end{tabular}

Table 1: Main ensemble parameters

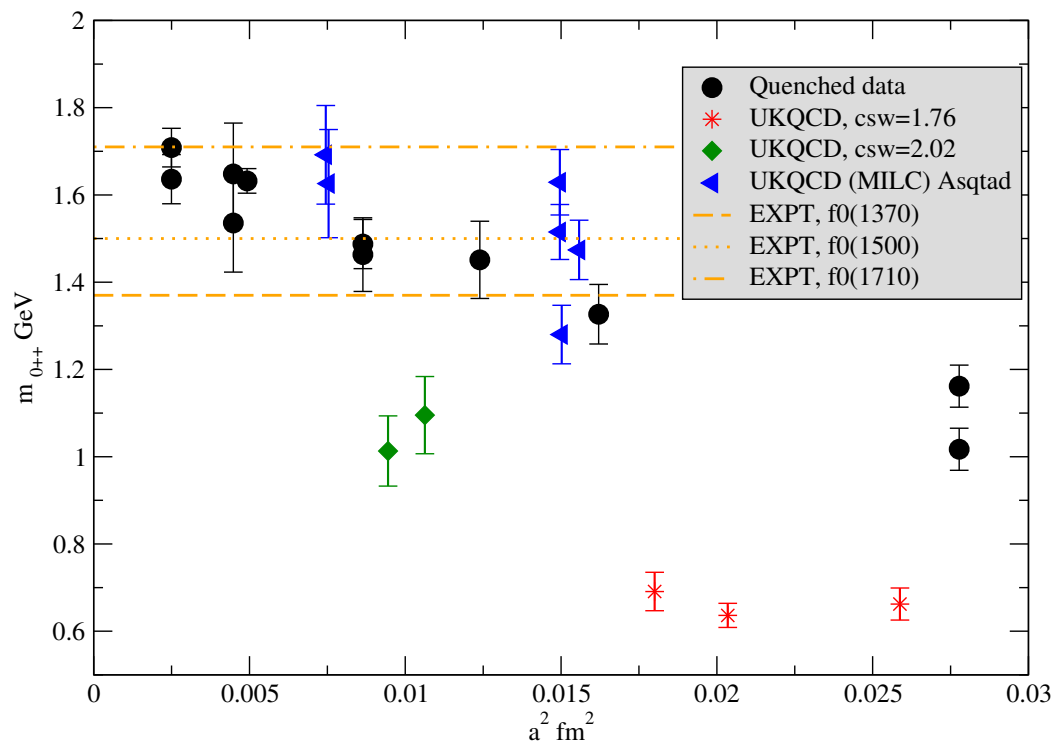

Figure 2: Compilation of glueball masses for quenched and unquenched QCD.

\section{Scalar mesons}

A staggered operator which creates a state that lies in the spin-taste representation $\Gamma_{S} \otimes \Gamma_{T}$ also couples to one lying in the $\gamma_{4} \gamma_{5} \Gamma_{S} \otimes \gamma_{4} \gamma_{5} \Gamma_{T}$ representation. Thus a staggered meson correlator has the general form

$$
\mathscr{C}(t)=\sum_{n}\left[A_{n} e^{-m_{n}\left(\Gamma_{S} \otimes \Gamma_{T}\right) t}+(-1)^{t} B_{n} e^{-m_{n}\left(\gamma_{4} \gamma_{5} \Gamma_{S} \otimes \gamma_{4} \gamma_{5} \Gamma_{T}\right) t}\right]
$$

In general, one therefore expects an oscillating contribution from a parity partner of the desired state. For the scalar meson $\left(\Gamma_{S} \otimes \Gamma_{T}=1 \otimes 1\right)$, the parity partner is $\gamma_{4} \gamma_{5} \otimes \gamma_{4} \gamma_{5}$ which corresponds to one of the pseudoscalars. For the non-singlet scalar this is one of the (taste-split) pions and so is a significant low lying contribution which must be included in the fits.

For flavour singlet mesons, the correlator is of the form

$$
\mathscr{C}(t)=C(t)-n_{f} D(t)
$$

where $C(t)$ is the same correlator coupling to the NS meson state and $D(t)$ is the disconnected (quark loops) correlator suitably corrected ( by $1 / 4$ ) to account for the extra 4 tastes that can 


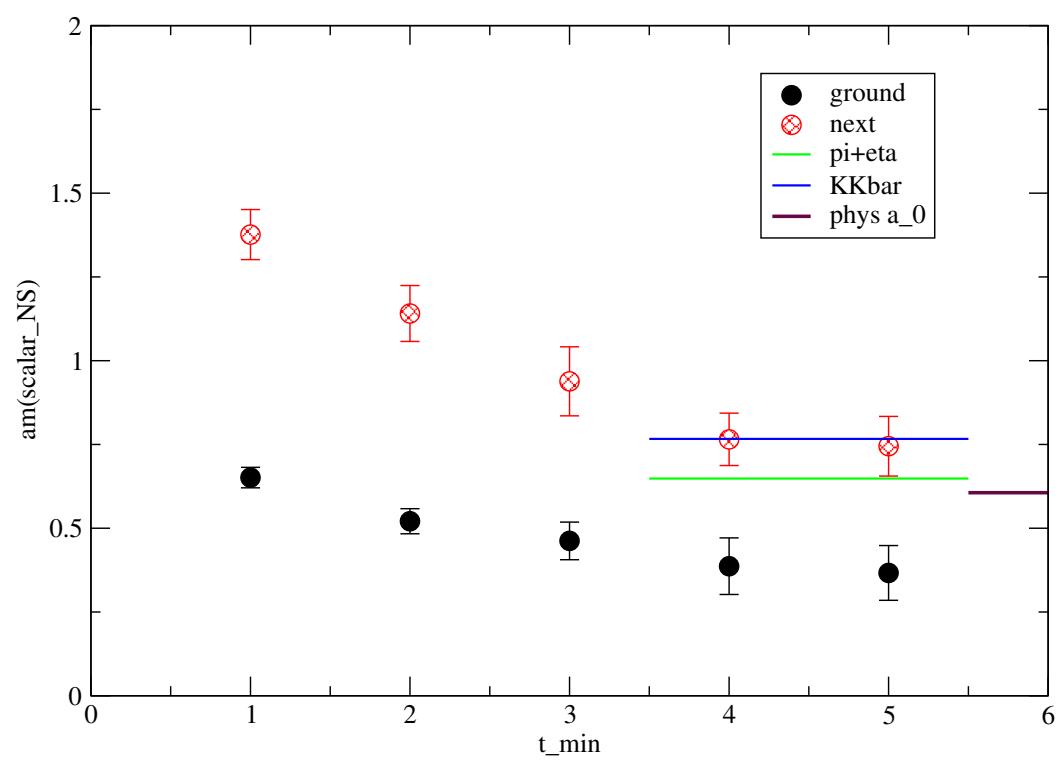

Figure 3: Non-singlet scalar mass as a function of minimum $t$ for ensemble $C$.

contribute as compared with the connected correlator [5]. In this study $n_{f}=2$ and the $u, d$ quark masses are degenerate. Note that there is no oscillating contribution to $D(t)$ in (3.1) since, in this case, the parity partner would be taste non-singlet [6].

\section{Non-singlet meson}

Figure 3 illustrates (for ensemble $C$ ) the resulting scalar masses from factorising fits to eqn. (3.1) using local and fuzzed operators. Three states were included - two scalar states and one oscillating pion. The plot shows the effect of varying the minimum $t$-value used in the fits. Here, $t_{\max }$ was fixed at 11 in lattice units. The most noticeable feature is that the 'ground state' estimate lies significantly below the effective $m_{\pi}+m_{\eta}$ threshold expressed in lattice units. Here the $\eta$ mass is estimated via the Gell-Mann Okubo mass formula following [3, \#] and the pion is taken to be the taste-singlet Goldstone pion.

The Figure also shows the $K \bar{K}$ threshold along with the observed mass of the $a_{0}(980)$ expressed in lattice units. This puzzling low-lying state was also observed by MILC authors [ $₫$ using independent measuring techniques - a different choice of operators and correlators.

There are a number of other remarks to be made concerning these fits and corresponding mass estimates. For this ensemble ( $\mathrm{C}$ - the lightest quark mass) the higher state appears roughly compatible (but possibly coincidentally) with the expected $a_{0}(980)$ mass and, hence with the nearby $K \bar{K}$ two-particle threshold estimated at this lattice spacing.

The oscillating parity partner state (pion) is well determined and consistent with direct measurement using a pseuoscalar operator. Qualitatively simliar behaviour is seen in the other ensembles $(A$ and $B)$. The fits did not require an excited oscillating state. Fits where the lowest state was constrained to match the expected mass of a $\pi+\eta$ state were unacceptable.

We recall that partially quenched studies of the NS scalar meson in other formulations show abnormal correlator behaviour [7]. The present study could be regarded as partially quenched in 


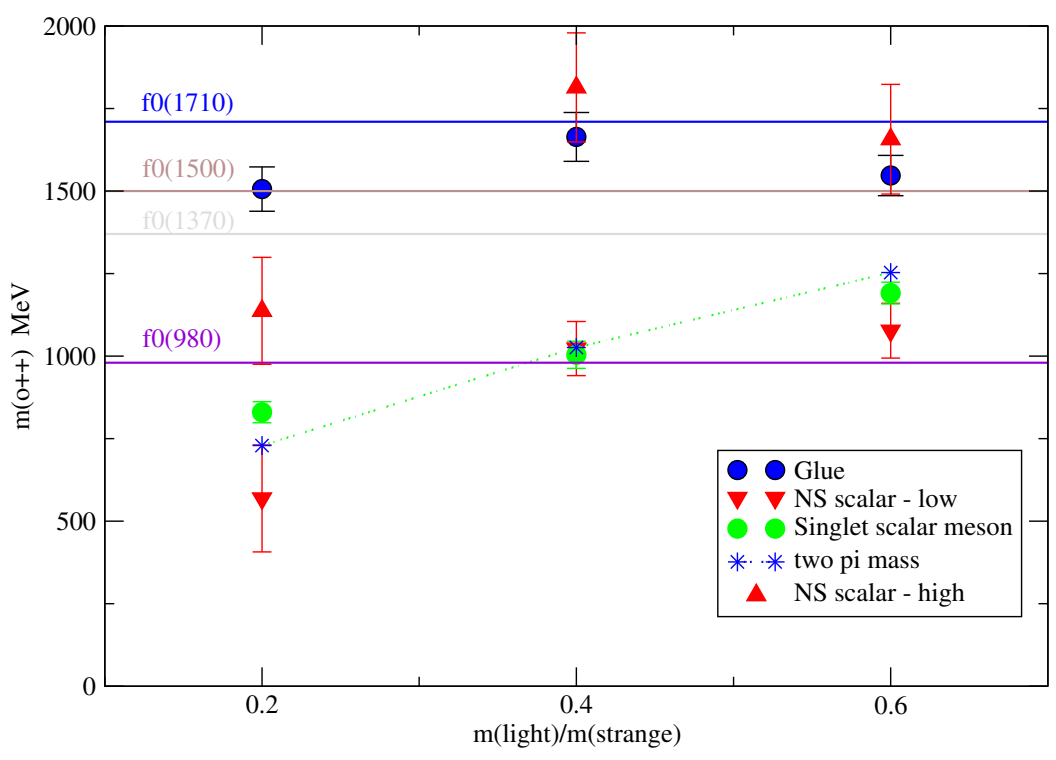

Figure 4: Singlet scalar mass as a function of quark mass.

the sense that the valence and sea quarks are treated differently with respect to taste - explicitly for valence operators but via the fourth-root trick for the sea.

Direct measurements of taste violations in the scalar NS sector are found to be comparable with those found in the pion sector. The maximum violation was of order $15 \%$ on the coarse lattice ensemble $A$. See [6] for details.

Prelovsek, in these proceedings [8], has used chiral perturbation theory for staggered fermions to show how taste violations in this channel can lead to effective G-parity violation in $a_{0}$ decay so that a small coupling to $\pi \pi$ is allowed. This is then expected [8] to dominate the correlator at large Euclidean time and so could account for the behaviour seen in Figure 3 and by MILC [ 4 ].

\section{Singlet scalar with light quarks}

The quark loops $L(t)=\operatorname{Tr} M^{-1}(\mathbf{x}, t)$ required for $D(t)=<L^{*}(t) L(0)>_{c}$ were evaluated using stochastic methods (with typicaly 48 noise vectors). Factorising fits, with local and fuzzed operators, were carried out in which the oscillating term in $C(t)$ was fixed from the $N S$ fits described earlier. The singlet scalar mass extracted in this way is shown as a function of the light quark mass in Figure $\theta$ (green circles).

For comparison we show again the masses obtained with glueball operators along with the corresponding $\pi \pi$ two particle energies. We also show the NS masses to illustrate the impact of the disconnected correlator contribution $D(t)$. One notes that the low-lying NS state does indeed track the $\pi \pi$ state at light quark masses [8].

With respect to the singlet meson operator fits, we note that useful signals for $D(t)$ were obtained out to $t=7,9$ and 10 for ensembles $A, B$ and $C$ respectively. Stable fits with one scalar and one (fixed) oscillating state were obtained with $t$ in the range 3-8 or so. We were not able to determine an excited scalar state. Since glueball correlators on these data sets were only well 
determined out to $t=3$ or 4 , the prospects of performing fully factorising fits incorporating both glueball and meson operators are not good with this level of statistics.

Unlike the NS case, the lowest energy singlet state with light quarks $(\bullet)$ lies above or close to the effective two particle threshold $(\star)$. In this case the $\pi \pi$ decay channel is physically allowed. In the flavour singlet case, the valence operator coupling to an $f_{0}$ state should include the strange quark. If included, this could well raise the ground state further above this threshold. Even with the correct strange quark content, the singlet state $(\bullet)$ is likely to lie below the non-singlet $(\triangle)$.

\section{Conclusions}

When improved staggered fermions are used to represent light dynamical quarks, the glueball mass is not significantly different from the quenched value at finite lattice spacing. Moderate taste violations are present in the scalar sector comparable with those observed for the pion. At light quark masses, decay channels complicate the mass determinations as do the effects of taste breaking in intermediate states. There is some evidence that the 2 flavour singlet meson lies below the corresponding non-singlet meson. Further details may be found in [6].

\section{Acknowledgements}

We would like to thank R Edwards and B Joo for assistance in code development with the SCIDAC-supported code Chroma [9], and Sasa Prelovsek for sharing her recent work on staggered chiral perurbation theory.

\section{References}

[1] TXL Collaboration, G. S. Bali et. al., Static potentials and glueball masses from qcd simulations with wilson sea quarks, Phys. Rev. D62 (2000) 054503, hep-lat/0 003012 .

[2] UKQCD Collaboration, A. Hart and M. Teper, On the glueball spectrum in o(a)-improved lattice qcd, Phys. Rev. D65 (2002) 034502, hep-lat/0108022.

[3] C. W. Bernard et. al., The qcd spectrum with three quark flavors, Phys. Rev. D64 (2001) 054506, hep-lat/0104002.

[4] C. Aubin et. al., Light hadrons with improved staggered quarks: Approaching the continuum limit, Phys. Rev. D70 (2004) 094505, hep-lat/ 0402030 ].

[5] L. Venkataraman and G. Kilcup, The eta' meson with staggered fermions, hep-lat/9711006

[6] S. Miller, A study of glueballs and scalars in lattice qcd, Univeristy of Liverpool Thesis (2005).

[7] S. Prelovsek, C. Dawson, T. Izubuchi, K. Orginos, and A. Soni, Scalar meson in dynamical and partially quenched two-flavor qcd: Lattice results and chiral loops, Phys. Rev. D70 (2004) 094503, hep-lat/0407037.

[8] Prelovsek, Effects of partial quenching and staggered fermions on scalar correlator, POS(LAT2005)085.

[9] SciDAC Collaboration, R. G. Edwards and B. Joo, The chroma software system for lattice qcd, hep-lat/0409003. 